\title{
Recurrent Childhood Rhabdomyosarcoma
}

National Cancer Institute

\section{Source}

National Cancer Institute. Recurrent Childhood Rhabdomyosarcoma. NCI Thesaurus.

Code C7815.

The reemergence of childhood rhabdomyosarcoma after a period of remission. 